\title{
A Spectroscopic Study of Be stars in the SMC Cluster NGC 330
}

\author{
W. Hummel, W. Gässler, B. Muschielok \\ Institut für Astronomie und Astrophysik und Universitätssternwarte \\ München, Scheinerstr 1, D-81673 München, Germany \\ T. Szeifert ${ }^{1}$, W. Seifert, I. Appenzeller \\ Landessternwarte Heidelberg, Königsstuhl 12, D-69117 Heidelberg, \\ Germany \\ G. Rupprecht \\ ESO, Karl-Schwarzschildstr. 2, D-85745 Garching, Germany
}

\begin{abstract}
We present low resolution spectroscopy of about 20 Be stars in the SMC cluster NGC 330 to confirm the high contents of Be stars in this cluster.
\end{abstract}

\section{Introduction}

The young open SMC cluster NGC 330 shows the highest number ratio of $\mathrm{Be}$ stars $(B e /(B e+B) \simeq 50 \%)$ and is known for its low metallicity. Comparison between galactic Be stars and Be stars in the field of NGC 330 could play a key role in constraining the conditions for the Be phenomenon in general. Spectra of the brightest Be stars in NGC 330 have been studied by Mazzali et al. (1996) and Keller \& Bessell (1998). In this study we analyze low resolution spectra of about 20 Be stars $\left(V=14^{\mathrm{m}}-19^{\mathrm{m}}\right)$ in the surrounding field of NGC 330 .

Observations have been collected on 3 nights during commissioning 1 of FORS1 at the VLT. The $\log$ of observations is given in Table 1. H $\alpha$ profile shapes are shown in Fig. 1.

\section{Results}

Among the $20 \mathrm{Be}$ stars selected from the list of photometrically identified Be stars of Grebel (1995) 18 could be confirmed to be classical Be stars. Hence we confirm the high contents of Be stars in NGC 330. Two of the fainter targets (Be 108 and Be 111) lack of emission though they have been identified as an $\mathrm{H} \alpha$ emission line object by Grebel (1995). Two targets have been observed twice (Be 68 and $\mathrm{Be} 55$ ) and no variability could be detected on the timescale of weeks.

${ }^{1}$ ESO Santiag, Alonso de Cordova 3107, Casilla 19001, Santiago 19, Chile 

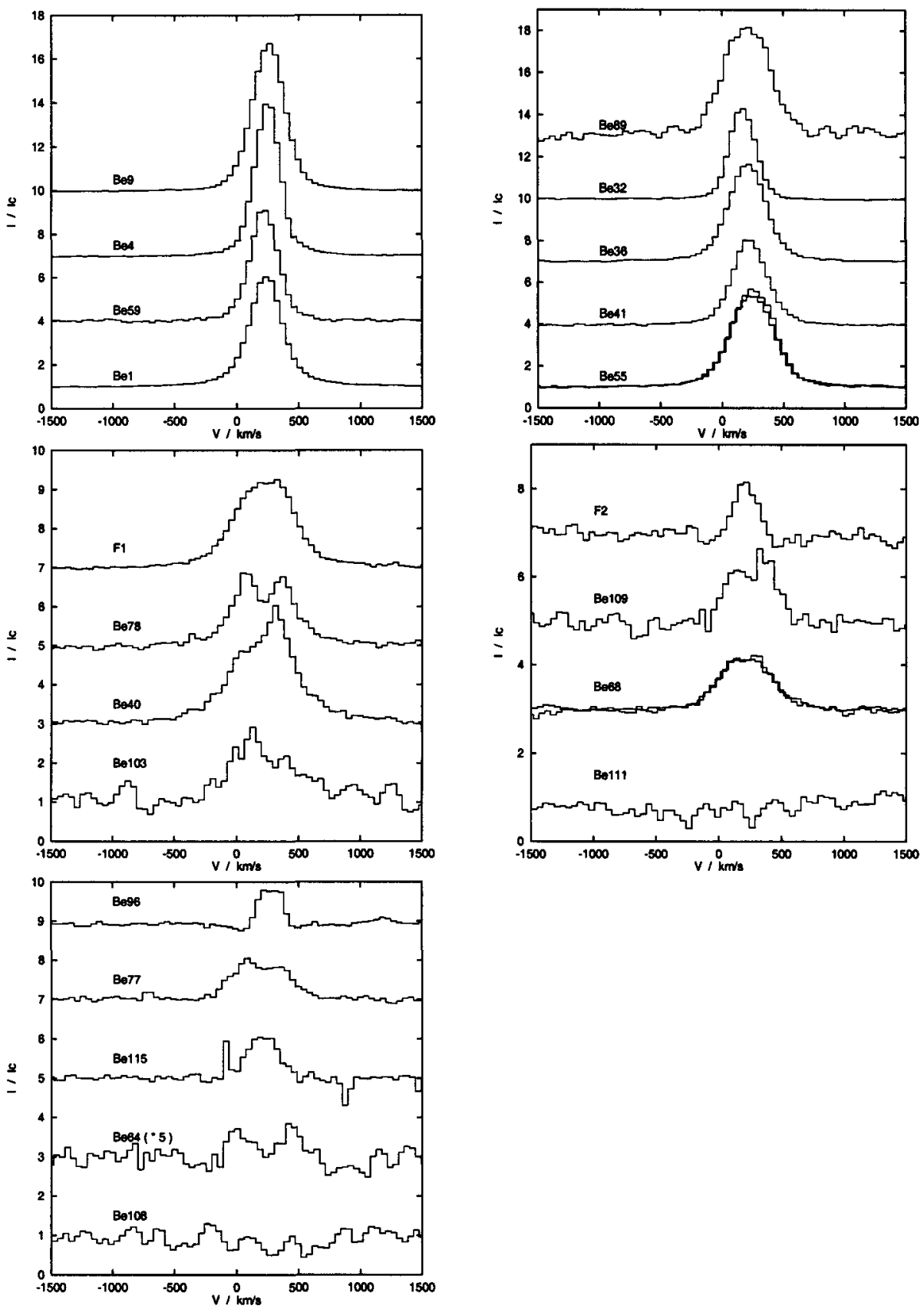

Figure 1. $\mathrm{H} \alpha$ emission line profiles of $\mathrm{Be}$ stars in the field of NGC 330 . Profiles are normalized to the local stellar continuum. The abscissa gives the radial velocity scale in the heliocentric frame. 

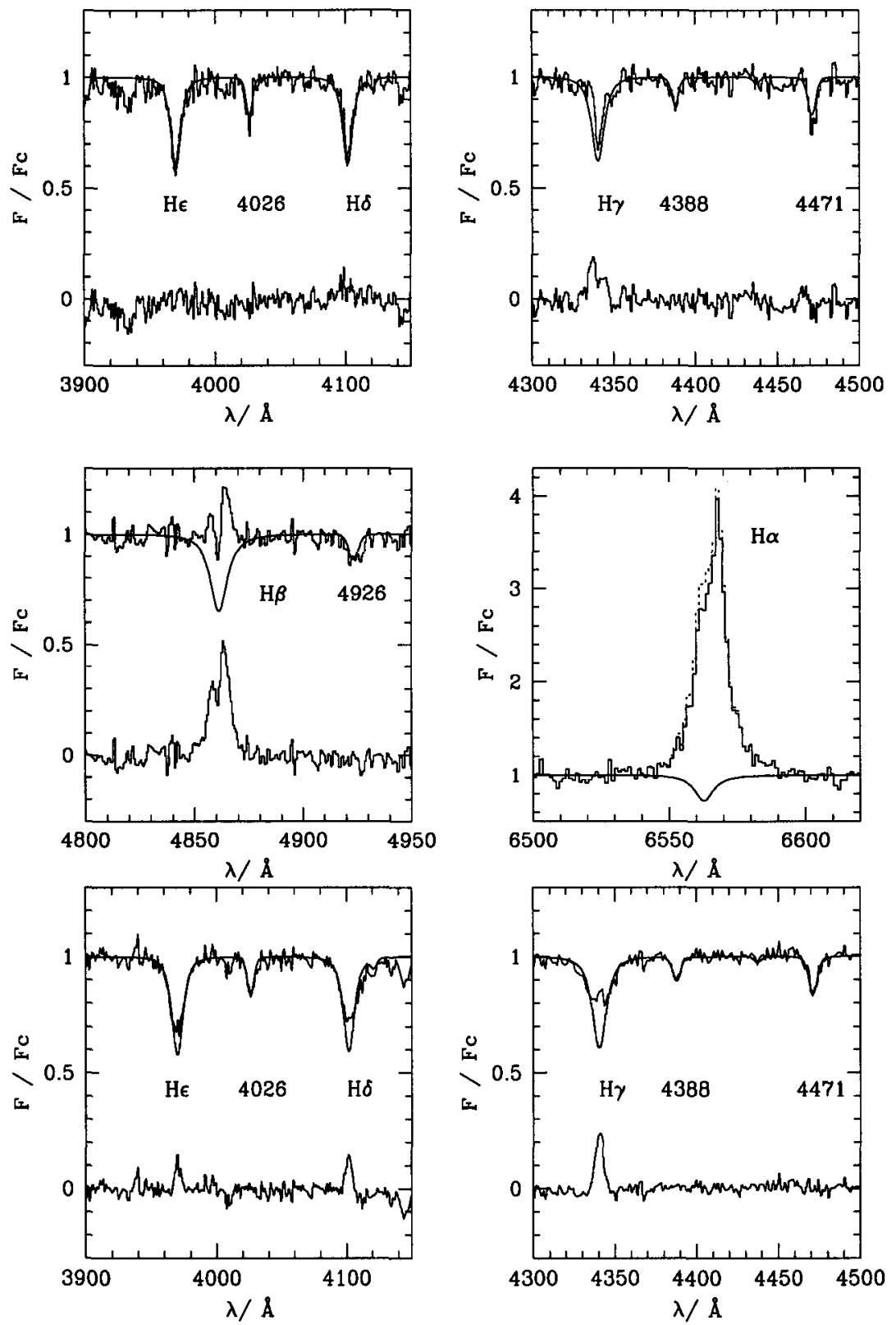

Figure 2. Best fit model and blue spectrum of Be 40 (upper four panels) and of Be 59 (lower two panels). The lower spectrum in each plot shows the circumstellar net emission. 
Table 1. Log of observations. From left to right: Julian date, date and UT, instrument mode, grism, filter and central wavelength, exposure time, seeing and background counts per pixel and second.

\begin{tabular}{cccccccccc}
\hline $\begin{array}{c}\text { JD } \\
+2451000\end{array}$ & $\begin{array}{c}\text { date } \\
1998\end{array}$ & UT & mode & grism & filter & $\begin{array}{c}\lambda_{\mathbf{c}} \\
\mathrm{nm}\end{array}$ & $\begin{array}{c}\text { time } \\
\text { sec }\end{array}$ & $\begin{array}{c}\text { seeing } \\
\text { " }\end{array}$ & $\begin{array}{c}\text { background } \\
\text { counts sec }^{-1}\end{array}$ \\
\hline 83.34 & $27 / 09$ & $06: 26$ & IMG & none & none & & 0.25 & 0.8 & 80 \\
88.28 & $02 / 10$ & $06: 43$ & MOS & $600 R$ & GG435 & 627 & 240 & 1.2 & 130 \\
88.28 & $02 / 10$ & $06: 52$ & MOS & $600 B$ & none & 462 & 360 & 1.2 & 130 \\
91.34 & $05 / 10$ & $08: 08$ & MOS & $600 B$ & none & 462 & 900 & 1.2 & 340 \\
91.35 & $05 / 10$ & $08: 37$ & MOS & $600 R$ & GG435 & 627 & 600 & 1.2 & 340
\end{tabular}

Two new Be stars (F1 and F2) have been detected in the field of NGC 330. F1 is out of the field studied by Grebel (1995) and appears at the southern rim of Keller et al. (1999). F2 is close to Be103 and has not been identified as an $\mathrm{H} \alpha$ emission line object by Grebel (1995) or Keller et al. (1999). Several targets (Be 78, Be 89, Be 77 and $\mathrm{Be} 108$ ) appear as close visual double stars on the preparation image. The resulting $\mathrm{H} \alpha$ line strengths are overestimated for these stars.

\section{Quantitative spectral analysis}

We fit model atmospheres of Vrancken et al. (1996) to several He I and Balmer absorption lines of Be $40\left(V=14^{\mathrm{m}} .41\right)$ and $\mathrm{Be} 59\left(V=17^{\mathrm{m}} .11\right)$. We used the method of Becker \& Butler (1990) to construct lines of constant EW in the $\log g-T_{\text {eff }}$ diagram. Best fit models and net emission are shown in Fig. 2. The resulting stellar parameters were $T_{\text {eff }}=18000 \pm 1500 \mathrm{~K}, \log g=3.3 \pm 0.2$ and $T_{\text {eff }}=17000 \pm 1500 \mathrm{~K}, \log g=3.1 \pm 0.2$ for Be 40 and Be 59 respectively. For Be 59 we could constrain $v \sin i$ to $150 \pm 40 \mathrm{kms}^{-1}$.

Acknowledgments. Based on observations collected during the commissioning of FORS1 at ESO, VLT observatory, Paranal, Chile. The funding through "Verbundforschung Astronomie" by the Bundesminister für Forschung, Bildung, Erziehung und Wissenschaft under grants 053MU104, 053GO10A and 053HD50A is gratefully acknowledged.

\section{References}

Becker, S., Butler, K., 1990, A\&A 235, 326

Grebel, E.K., 1995, PhD., University Bonn

Keller, S.C., Bessell, M.S., 1998, A\&A 340, 397

Keller, S.C., Wood, P.R., Bessell, M.S., 1999, A\&AS 134, 489

Mazzali, P.A., Lennon, D.P., Pasian, F., Marconi, G., Baade, D., Castellani, V., 1996, A\&A 316, 173

Vrancken, M., Butler, K., Becker, S., 1996, A\&A 331, 661 